\title{
Cost of managing an episode of relapse in multiple sclerosis in the United States
}

\author{
Judith A O'Brien*1, Alexandra J Ward ${ }^{1}$, Amanda R Patrick ${ }^{1}$ and Jaime Caro ${ }^{1,2}$
}

Address: ${ }^{1}$ Caro Research Institute, Concord, MA 01742, USA and ${ }^{2}$ Division of General Internal Medicine, McGill University, Montreal, H3A 2T5 Canada

Email: Judith A O'Brien* - jobrien@caroresearch.com; Alexandra J Ward - alexward@ caroresearch.com; Amanda R Patrick - apatrick@hsph.harvard.edu; Jaime Caro - jcaro@caroresearch.com

* Corresponding author

Published: 02 September 2003

BMC Health Services Research 2003, 3:17
Received: 02 June 2003

Accepted: 02 September 2003

This article is available from: http://www.biomedcentral.com/I472-6963/3//7

(C) 2003 O'Brien et al; licensee BioMed Central Ltd. This is an Open Access article: verbatim copying and redistribution of this article are permitted in all media for any purpose, provided this notice is preserved along with the article's original URL.

\begin{abstract}
Background: The purpose of this study was to determine the direct medical US cost of managing multiple sclerosis relapses.

Methods: Direct data analysis and cost modeling were employed to derive typical resource use profiles and costs in 2002 US dollars, from the perspective of a third-party payer responsible for comprehensive health-care. The location and scope of health care services provided over a 90 -day period were used to define three levels of relapse management. Hospitalization and resulting subsequent care was defined as high intensity management. A medium level of intervention was defined as either use of the emergency room, an observational unit, or administration of acute treatments, such as intravenous methylprednisolone in an outpatient or home setting. The lowest intensity of care comprised physician office visits and symptom-related medications. Data were obtained from many sources including all payer inpatient, ambulatory and emergency room databases from several states, fee schedules, government reports, and literature. All charges were adjusted using cost-to-charge ratios.
\end{abstract}

Results: Average cost per person for high management level was $\$ 12,870$, based on analysis of 4,634 hospital cases (mean age 48 years, $73 \%$ female). Hospital care comprised $71 \%$ of that cost. At discharge, $36 \%$ required inpatient sub-acute care, rehabilitation or home care. The typical cost per moderate episode was $\$ 1,847$ and mild episode $\$ 243$.

Conclusions: Management strategies leading to a reduction in the frequency and severity of a relapse, less reliance on inpatient care, or increased access to steroid infusions in the home, would have a substantial impact on the economic consequences of managing relapses.

\section{Background}

Although the exact number of people with multiple sclerosis (MS) is not known in the United States (US), published estimates put the number at between 250,000 to 350,000 [1]. The majority of patients initially have a form of MS described as "relapsing-remitting", which manifests as a series of exacerbations followed by periods of partial or complete remission, and $30-50 \%$ of those will have progressive symptoms within the first ten years [2]. Relapse rates vary widely ( 0.1 to 1 attack per year), and patients with higher relapse rates in the first two years after onset of symptoms have been observed to be at 
greater risk of more rapid progression to a severe level of disability [3]. Most people experience their first symptoms between the ages of 20 and 40 years; therefore, this disease may have a substantial impact over many years on their health, quality of life, productivity and employment $[1,4]$. The annual cost of MS in the US has been estimated to be $\$ 2.5$ billion [1].

In a clinical area where relief of symptoms has been the mainstay of management, the development of new disease modifying drugs for MS in recent years has provided hope for patients due to their important clinical benefits. Data published by the National Consortium of Multiple Sclerosis Centers in 2000, [5] showed that $78 \%$ of patients questioned reported ever taking a disease modifying drug and $67 \%$ of patients were currently taking one. Clearly, these agents have been embraced as a therapy for relapsing-remitting MS and in a cost conscious clinical environment concerned with limited health care dollars their impact should be examined not only in clinical terms such as reducing relapse rates, or severity or modifying the progression of the disease, but also with an understanding of their economic consequences. Medical decision-making in managed care organizations (MCO) and elsewhere should not take place in an economic vacuum. To facilitate these decisions, it is necessary to have current estimates of the direct medical costs related to managing MS. As there is little published in the literature relating to the direct medical cost of relapse in the U.S, this paper focuses on providing a key piece of the economic puzzle for relapsing-remitting MS - the cost of managing an episode of relapse.

\section{Methods \\ Study Design}

The goal of this analysis is to provide estimates of the direct medical costs of managing a relapse in multiple sclerosis. These costs are meant to reflect the economic value of the resources consumed, regardless of who actually pays for them (for example, Medicare, Medicaid or a commercial insurer). Thus, they do not take into account adjustments made in the multi-payer US system, such as differences in coverage of medications, co-payments, reimbursement rates or volume discounts.

The cost estimates were developed to provide a comprehensive picture of the direct medical costs associated with managing a relapse of MS. The range of health care services used to manage a relapse will consequently reflect the severity of the initial signs and symptoms. When patients have a relapse, they can experience a diverse range of symptoms of neurological dysfunction, which eventually may resolve partially, completely, or stabilize [6]. The severity of symptoms experienced during a relapse varies widely and may include sensory loss, optical neuritis, weakness of the limbs leading to fatigue, disturbance of gait, loss of dexterity, and paraesthesias [6]. The duration of a relapse episode also varies and may last up to several months[6].

Symptoms associated with a relapse can be treated in a number of ways and at different levels of formal care. Therefore, for the purpose of this analysis, the place where health care was delivered and scope of services provided was used to define three levels of management of a relapse. The health care services and their associated costs used during a relapse episode, defined as a 90-day period for this analysis based on information from the literature [7], were estimated for each of the three management levels. Hospitalization was defined as high intensity management of a relapse. A medium level of intervention was considered to be either use of the emergency room (ER), or an observational unit, or administration of acute treatments requiring formal intervention, such as intravenous (IV) methylprednisolone given in an outpatient or home setting. The lowest intensity of care provided for a relapse is comprised of physician office visits and symptomrelated medications. The major cost centers included in the profiles for each management level are indicated in Figure 1.

As there is no single data source available that provides all of the information required to derive the cost of a relapse, it was necessary to gather data from a variety of sources and employ a combination of direct data analysis and cost modeling based on typical clinical management profiles. A resource use profile for each management level was developed by identifying the health care goods and services relevant to a relapse, quantifying the frequency of use and proportion of users for each item included in the profile, and applying a unit cost to each resource used.

\section{Data Sources}

\section{Hospital inpatient care}

Inpatient resource use and costs were derived from an analysis of all payer, 1999 discharge data from five states representing different areas of the US (California, Florida, Maryland, Massachusetts, Washington) [8-12]. These databases include demographic, clinical, and economic data for discharges from 980 hospitals. Patients diagnosed with MS were identified in each database (principal or secondary diagnosis International Classification of Diseases code, ICD-9 code: 340 ). When MS was coded as a secondary diagnosis, only those cases with a principal diagnosis of a condition or symptom that could be associated with a relapse were included in the analyses. All cases with a principal diagnosis of depression were excluded from the analysis. 


\section{Relapse Cost Components}

\section{Health Care Services in Resource Use Profiles}

\section{Low Intensity Episode}

- Initial Contact:

- Usual care MD

- Symptom-Related Meds

- Follow-up Office Visits
Moderate Intensity Episode

- Initial Contact:

- Usual care MD

- Emergency Room

- IV Methylprednisolone

- Hospital day case

- Home administration

Symptom-Related Meds

- Follow-up Office Visits

- Consults

Therapist

- Physical

- Occupational

- Speech
High Intensity Episode

- Initial Contact:

- Usual care MD

- Emergency Room

- Hospital Admission

- All acommodations

- All ancillaries

- Physician care

- Post-Discharge Services

- Outpatient follow-up

- Rehabilitation

- Home health care

- Skilled nursing facility

- Short stay nursing home care

- Hospital readmission within 30 days

Figure 1 Overview of health care services and resource use profiles used to define management levels during a 90-day relapse episode

(Abbreviation: $\mathrm{IV}=$ intravenous, $\mathrm{MD}=$ medical doctor)

Figure I

Hospital costs include all accommodations, all ancillary and physician-related services. ER costs are included for those who used that service prior to admission. Inpatient physician care profiles were developed for cases of MS from the information in the five hospital discharge databases. These profiles were developed based on the recorded length of stay (LOS), procedure codes, special care unit and ER use, surgical and anesthesia activities, consultations and other physician-related database ele- ments. For patients 65 years and older, the 2002 Medicare national physician fee schedules [13] were applied to estimate the cost of inpatient and outpatient physicianrelated services. For younger patients, the Medicare fees were adjusted with ratios derived from published fees for common types of insurers (i.e. Medicaid, preferred provider organizations, and indemnity plans) [14,15]. This methodology was applied to all physicians and test costs at all management levels in this analysis. 


\section{Post-acute care}

Health care service use does not end upon discharge from the hospital; therefore, care after hospitalization was factored into the management profiles for those who required hospital level of care. The location of care (i.e., discharged home to outpatient care or with home health care services, transferred to rehabilitation units, skilled nursing facilities (SNF), or medically supervised nursing homes for short-term care) following discharge was determined using disposition status data from the five states. In addition, re-admission to the acute care hospital for a similar problem within one month of the index admission was considered to be a component of the costs of the relapse episode. Readmission patterns were analyzed using the data from the Massachusetts database, which includes unique patient identifiers [10].

The length of stay and costs of inpatient rehabilitation care were estimated from the same five state databases. The cases were selected where the primary reason for admission was rehabilitation and MS was coded as a secondary diagnosis. The disposition status at discharge from inpatient rehabilitation was examined to determine if the patient returned home, required home health care service or was transferred to a skilled nursing facility. These data were supplemented with information published by the New Hampshire Hospital Association on patients admitted with MS to rehabilitation facilities for the years 1996 through 1999 [16-19]. Medicare and Medicaid data published by the Center for Medicare and Medicaid Services (CMS) were used to establish the LOS, visit rate and unit cost information for SNF care and home health care services [20]. Nursing home per diem costs were established by analyzing statewide data for patients with a primary diagnosis of MS when admitted to long term care facilities in Minnesota [21]. The Minnesota costs were adjusted to national values using published nursing home per diem costs [22]. The rate of physician visits to the nursing home was estimated from medical literature [23].

\section{Emergency Room and Observation Unit Visits}

Estimates of resource use and cost data were obtained from the 1999 Utah all payer ER database [24] and the 1999 Massachusetts Observation Unit database [25]. The criteria used for selecting MS cases were the same as those used for inpatient care.

\section{Outpatient Care}

Data from the 1999 Florida Medicaid ambulatory care database [26] and the National Ambulatory Care Survey (NAMCS) [27-29], as well as published Medicare and Medicaid data $[13,14]$ and published literature [30-34] were examined and used to develop the outpatient profiles and management costs. In addition, NAMCS data from the 1997, 1998, and 1999 databases [27-29,35] were pooled and weighted by patient visit weights as described in the National Ambulatory Medical Care Survey Data File Documentation [36]. The NAMCS is a probability sample where data are derived from a randomly selected group of office-based physicians [37]. In those with a coded diagnosis of MS, the general reason for the physician encounter was used to discern a routine visit for a chronic condition (assumed to be a remission-related usual care visit) from a visit to the physician for an acute problem, which was assumed to be a relapse-related visit. The disposition status at the end of the visit was used to determine whether patients were scheduled for a followup visit, referred to a consultant, not scheduled to return or those who should return only if necessary.

Basic laboratory tests such as a complete blood count (CBC) and basic metabolic profile were included in the cost of the initial comprehensive assessment visit with the usual care physician. The proportions of patients receiving other tests were estimated from NAMCS data and the literature [32]. All patients were assigned the cost of one magnetic resonance imaging (MRI) scan. Resource use and costs for relapse-related outpatient treatment for physical therapy (PT), occupational therapy (OT) and speech therapy (ST) were obtained from NAMCS data, CMS data and the literature $[14,15,27-29,32]$. The unit cost for an initial comprehensive medical assessment visit, follow-up office visit with the usual care physician, and consultation with specialists was established using the appropriate CPT-4 code [38], the Medicare physician and laboratory fee schedules for those $\geq 65$ years and the derived unit costs for those $<65$ years based on the methodology described previously.

\section{Medications}

The daily cost of outpatient medications prescribed to manage symptoms during a relapse episode (e.g., amitriptyline, baclofen) was included in the cost estimates derived for each level of management. The proportion of patients receiving medication to manage symptoms during a relapse and the daily cost was estimated from the literature and the 2001 Red Book $[5,30,39,40]$. The cost of disease modifying drugs such as interferon- $\beta-1 b$ (Betaseron), interferon- $\beta-1 \mathrm{a}$ (Avonex) and glatiramer acetate/copolymer 1 (glatiramer, Copaxone) were considered to be ongoing management costs and were excluded from the costs associated with a relapse. Intravenous (IV) methylprednisolone followed by a tapering dose of oral prednisone is used to treat acute exacerbations $[31,33,41]$. The frequency of use of oral steroids by those receiving infusions was determined from the literature [31]. For those receiving inpatient management, the cost of methylprednisolone infusions is included in the hospital stay cost estimates, but as this treatment is also administered in the outpatient setting, 
Table I: Mean unit costs by major cost categories. Overall costs are weighted average of component costs applied to the proportion of patients using the service. Thus, the individual components will not add up to the overall cost

\begin{tabular}{|c|c|c|c|}
\hline Management level & Cost component & Unit & Mean Unit Cost 2002 US \$ \\
\hline \multirow[t]{3}{*}{ Low } & Overall cost & Per episode & 243 \\
\hline & Physician visits & Per episode & 170 \\
\hline & Symptom-related medications & Per episode & 73 \\
\hline \multirow[t]{10}{*}{ Medium } & Overall cost & Per episode & 1,847 \\
\hline & ER visit & Per visit & 475 \\
\hline & Observation unit & Per stay & 2,111 \\
\hline & Initial office visit for acute problem with $\mathrm{CBC}$, BMP & Per visit & 240 \\
\hline & IV Methylprednisolone: & & \\
\hline & Day case & Per episode & 3,184 \\
\hline & Home care & Per episode & 792 \\
\hline & Symptom-related medications & Per episode & 73 \\
\hline & Other diagnostic and laboratory tests & Per episode & 849 \\
\hline & Therapies (i.e., PT, OT, ST) & Per episode & 207 \\
\hline \multirow[t]{8}{*}{ High } & Overall cost & Per episode & 12,870 \\
\hline & Acute hospital & Per stay & 8,782 \\
\hline & Post-discharge & Per episode & 4,088 \\
\hline & Rehabilitation facility & Per stay & 14,943 \\
\hline & Skilled nursing facility & Per stay & 10,514 \\
\hline & Short term nursing home & Per stay & 12,894 \\
\hline & Home heath care services & Per episode & 2,043 \\
\hline & Outpatient care & Per episode & 114 \\
\hline
\end{tabular}

(Abbreviations: $\mathrm{ER}=$ emergency room, $\mathrm{CBC}=$ complete blood count, $\mathrm{BMP}=$ basic metabolic profile, $\mathrm{PT}=$ physical therapy, $\mathrm{OT}=$ occupational therapy, ST=speech therapy)

this therapy was included in the cost of the medium intensity management level as well. The cost of administering infusions at home was obtained from the literature [42]. A day case cost was derived by analyzing a subset of the MS cases in the Massachusetts Observation Unit data set that received steroids by infusion [25]. To identify these cases, all the Observation Unit cases were screened for ICD-9 procedure codes 99.25 (injection of steroid) and 99.29 (injection or infusion of other therapeutic or prophylactic substance).

\section{Cost Estimates}

All cost estimates are reported in 2002 US dollars. Where 2002 values were not available, older estimates were inflated using rates based on the medical care component of the United States Consumer Price Index, supplied by the Federal Bureau of Labor Statistics [43]. Any charges used as inputs (e.g., inpatient hospital care) were adjusted to costs using a cost-to-charge ratio. In the absence of a standard value, a ratio of 0.61 was used, as this is the most recent figure calculated by the Commonwealth of Massachusetts Office of Health Care Finance and Policy for hospitals in Massachusetts. Where single state databases were used (e.g., ER, observation unit nursing home), state-specific costs were adjusted to national values using CMS geographical cost information [20].

\section{Results}

\section{High Level}

Data from the five state discharge databases revealed that a diagnosis of MS was recorded for 17,743 cases, and as a primary diagnosis in 3,421 (19\%) cases. Based on the study criteria, when MS was coded as a secondary diagnosis, only those cases with a principal diagnosis of a condition or symptom that could be associated with a relapse were included in the analyses $(1,213$ cases). A total of 4,634 study-eligible cases were identified. The majority (73\%) was female. The mean age was 48 years, and $54 \%$ of the cases were between the ages of 25 and 44 years.

Primary payer data revealed that managed care organizations (MCO) comprise the second largest payer category for inpatient care. For this analysis, the MCO payer category includes various types of non-Medicare and nonMedicaid funded health care insurance plans including health maintenance organizations (HMO), preferred provider organizations (PPO) and point of service plans (POS) (Figure 2). Of note, although only $10 \%$ of patients are $>=65$ years, Medicare is the responsible payer for $39 \%$ of the admissions and is the largest single payer. The high proportion of Medicare patients with MS in this sample is likely due to those who qualify for this type of coverage due to a disability rather than age. The Medicare category includes those covered under the traditional fee for service 
plan (83\%) and Medicare sponsored managed care plans

$(17 \%)$.

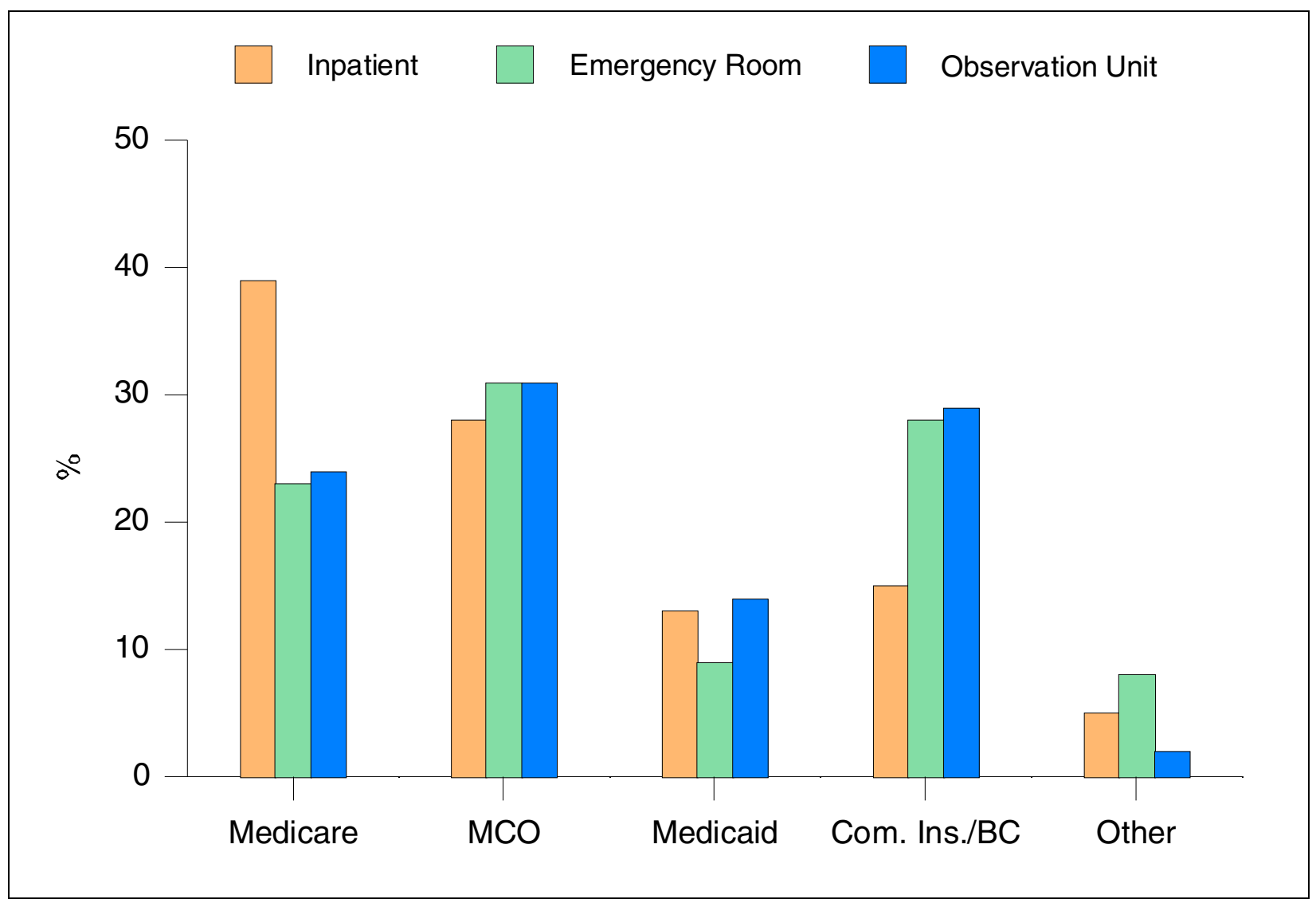

Figure 2 Distribution of multiple sclerosis cases from state databases by primary payer

(Abbreviations: MCO-managed care organization; Com Ins.=commercial insurance; BC=Blue Cross)

Figure 2

The mean LOS for a hospital stay was 5.2 days (range: $1-$ 353 ) with a mean cost of $\$ 8,782$ (range: $\$ 410-1,045,376$ ) when all cases are considered. If the study group is restricted to those in hospital 90 days or less, we would eliminate 15 cases $(0.32 \%)$ and the mean cost would be $\$ 8,392$ (range: $\$ 420-302,545$ ). As would be expected, acute care hospitalization is the major cost component of high-level management, accounting for $70 \%$ of the total cost. From our analyses, half of all cases were admitted via the ER. The patients who are seen in the ER prior to hospitalization have a higher cost per stay $(\$ 9,076)$ on aver- age than those referred to the hospital directly by their physician $(\$ 8,183)$. Table 1 provides a summary of costs by major cost category used in this analysis.

The inpatient case fatality rate was $1 \%$. Of those who survived the hospitalization: $59 \%$ were discharged to home; $14 \%$ with home health care services (mean cost of $\$ 2,043$ per relapse episode); $10 \%$ to inpatient rehabilitation (mean LOS $=15$ days, range: $1-126$ days; mean cost per stay $\$ 14,943$, range: $\$ 645-\$ 105,480) ; 11 \%$ to a skilled nursing facility (mean LOS $=28$ days, mean cost per stay 
$\$ 10,514)$; and $1 \%$ to nursing home care (mean cost per 90 day stay $\$ 12,894$, range: $\$ 7,709-\$ 14,200)$. The costs of these levels of care were applied proportionately to the users of those services to derive the typical overall weighted post-acute care cost. For the remaining 5\% (other), the disposition status was either unknown or was a level of care (e.g., custodial residential care) that would not be covered by third party payers in the US and is therefore not a direct medical cost relevant to this analysis. Of those discharged initially to a rehabilitation facility or unit, $1 \%$ died during their stay, $8 \%$ were transferred to a skilled nursing facility, 38\% required home health care services after discharge and the remainder were followed as outpatients.

For those discharged to home, follow-up outpatient care consisted of one physician visit (\$37), and the calculated weighted average cost of symptom related medications (\$73) for 90 days less the LOS in hospital. For patients discharged from a SNF or inpatient rehabilitation, the cost of an outpatient follow-up physician office visit care plus a home assessment visit (\$35) was added to the cost of their sub-acute stay. In addition, the cost of symptom related medications was added for the 90-day period minus the number of days the patients was in acute and sub-acute inpatient care. Furthermore, the cost of a repeat hospitalization was included in the post-acute care estimate for the $4 \%$ who were readmitted within 30 days of discharge. When all post-acute care costs are weighted proportionately by the percent of those who used them, the typical post-discharge subsequent care cost per person is estimated at $\$ 4,088$ (potential range: $\$ 37-\$ 24,382$ ) per person per 90-day relapse episode.

Thus, for patients who experience a relapse with symptoms severe enough to require hospitalization, the typical cost for a relapse episode over 90-days is $\$ 12,870$. Figure 3 provides an overview of the proportional contribution of the major cost components to the typical cost for this management level.

\section{Medium Level}

Separate resource use profiles were developed for those who were initially seen in the ER versus those whose initial encounter was with their usual care physician at the onset of symptoms. Based on 401 cases identified in the ER database, of whom $76 \%$ were female and $44 \%$ were between the ages of 25-44 years, the mean cost of an ER visit for those discharged to outpatient care after the visit was estimated at $\$ 475$ (range: $\$ 30-\$ 7,745$ ). This cost includes facility, ancillary and physician costs. The mean time for an ER visit was 7 hours (range: 1-23 hours) and a $\mathrm{MCO}$ was the responsible payer for $32 \%$ of the cases (Figure 2).
Analysis of the Massachusetts Observation Unit database resulted in identification of 369 study-relevant cases. Females comprised $68 \%$ of the cases and the mean age was 48 years. The mean observation time was 25.8 hours (range: 1-90 hours) with a mean cost per stay of $\$ 2,111$ (range:\$23-\$20,853). MCOs were the primary payer for almost a third of the observation unit cases (Figure 2).

The cost of administering methylprednisolone IV (750 mg per day over 8 hours for four days) was estimated both when provided as a day case $(\$ 3,814)$ and home care (\$792). The day case estimate was obtained by analyzing 30 cases attending Massachusetts Observation Units, who received IV steroids. In the absence of published information about the proportion of patients who receive infusions as observed day cases versus in a home care setting in the US, an assumption was made that $25 \%$ of the $56 \%$ [31] of relapse patients who receive this therapy are treated as day cases. The cost of a course of oral prednisone was estimated at $\$ 3.33$ and was applied to $21 \%$ [31] of the patients receiving infusions. The cost of the initial comprehensive office visit with the patient's usual care physician including basic laboratory tests was estimated at $\$ 240$. The proportions of patients receiving other tests on follow-up were as follows: $11 \%$ urinalysis, $4 \%$ ultrasound of bladder or bladder function studies, 2\% spinal tap, and $2 \%$ electromyography. Based on information from NAMCS the medium intensity cost for usual care physician follow-up and consults was derived based on $91 \%$ returning for a follow-up office visit, $10 \%$ referred to a urologist, $5 \%$ to an ophthalmologist and $12 \%$ to other specialists. The weighted cost of these additional tests, referrals and follow-up visits were applied to both the ER and usual care physician profiles. In addition, the cost of a course of outpatient physical $(\$ 1,117)$, speech $(\$ 681)$ and occupational therapy $(\$ 1,240)$ was applied to $10 \%$, $6 \%$ and $5 \%$ of patients respectively [5]. The cost of symptom related medications was applied to $95 \%$ of the patients [30] for 90 days to complete this profile.

For patients who see their usual care physician initially, the typical cost for a relapse episode that required this level of management was estimated to be $\$ 1,836$, and $31 \%$ higher for patients whose symptoms were evaluated initially in the ER $(\$ 2,408)$. This increase is due to the incremental cost of the ER visit plus physician services rendered in the ER for a serious but non-life threatening condition and a follow-up visit to the patient's usual care physician soon after the ER visit. Otherwise, the profiles are identical. Based on NAMCS data analyses, just $2 \%$ of the patients were initially seen in the ER, after weighting these costs appropriately, a relapse episode with this medium level of management costs $\$ 1,847$, on average. The low end of the range was estimated to be $\$ 316$ for those who do not receive IV methylprednisolone to 


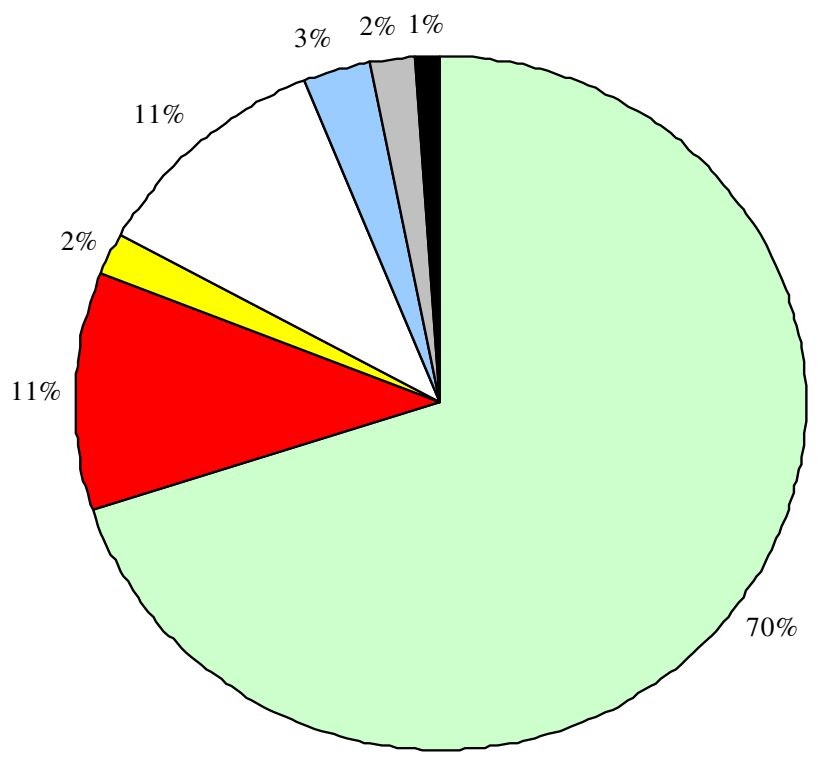

$\square$ Hospital Stay

Rehabilitation

Pre-Admission Emergency Room

$\square$ SNF Care

Short Stay Nursing Home Care

Home Health Care Services

Post-Discharge Outpt Follow-up

\section{Figure 3 Proportion of overall high-level management cost by major cost components}

(Abbreviations: SNF=skilled nursing facility, Outpt=outpatient)

\section{Figure 3}

$\$ 12,525$ when an ER visit is made followed by extensive testing and multiple referrals. Figure 4 provides an overview of the proportional contribution of the major cost components for the typical medium level management cost.

\section{Low Level}

The overall cost of a relapse that receives a low intensity level of management is estimated at $\$ 243$ (range: $\$ 171-$ $\$ 952)$ per person. The major cost component is the cost of the initial office visit with the patient's usual care physician including basic lab tests for those who receive them. Based on analyses of the NAMCS data $64 \%$ of patients return for a follow-up visit. As for the other profiles, the cost of medications to relieve symptoms during the relapse episode was applied to $95 \%$ of the patients based on information from the literature [30].

\section{Discussion}

Relapses and the progression of the disease with the development of irreversible disability are two key clinical characteristics of MS $[3,44,45]$. Acute exacerbations of MS are commonly managed with infusions of steroids, such as methylprednisolone administered in the out patient setting [46]. In fact, some insurers now have criteria that have to be met before a patient can be admitted overnight for this type of therapy [47]. Yet, there remain some cases where a relapse has to be managed intensively and requires an admission to hospital, for example, when a severe neurological deficit is present [47]. Previous studies have shown that hospitalization remains a significant 


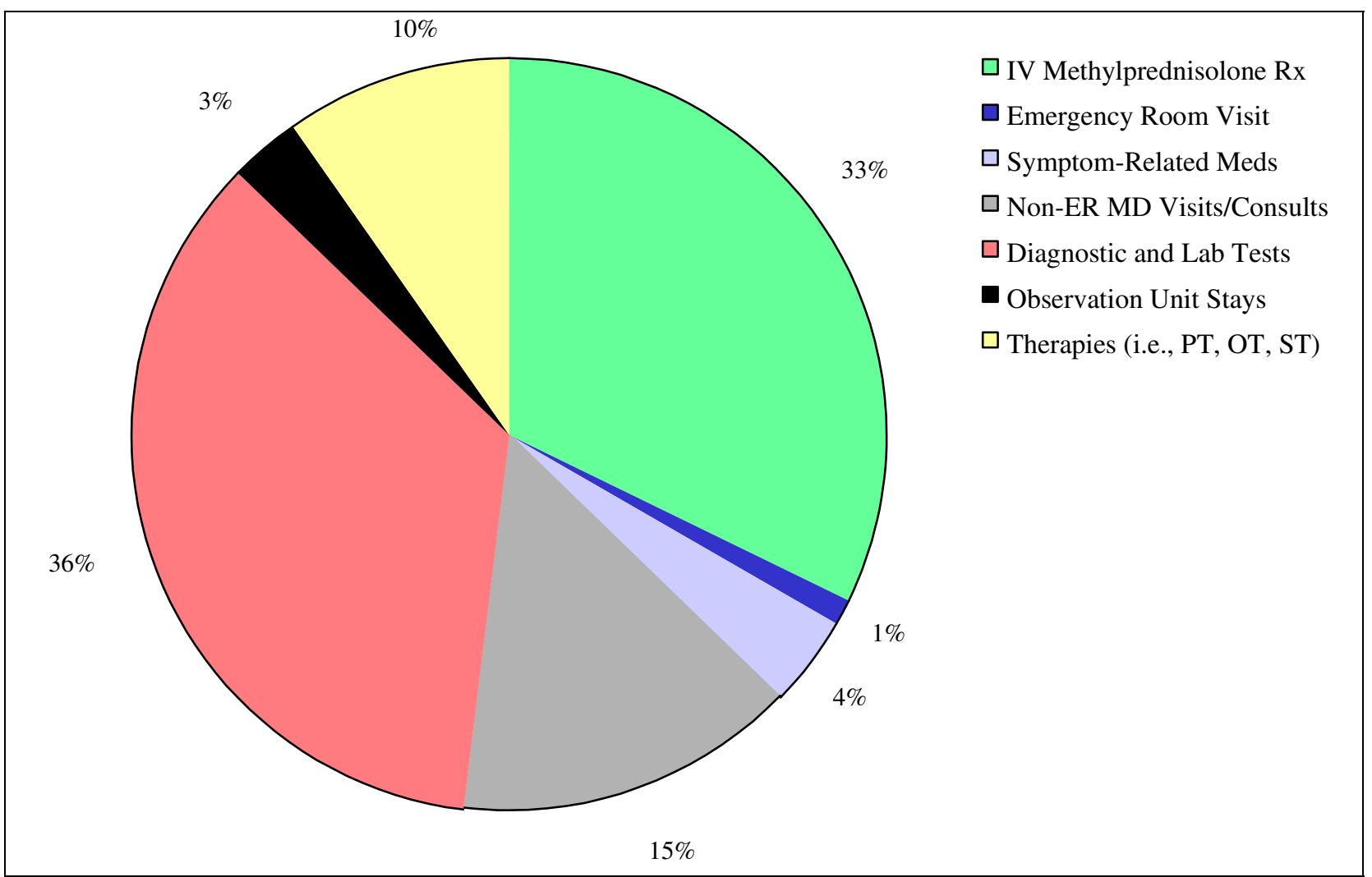

\section{Figure 4 Proportion of overall medium level management cost by major cost components}

(Abbreviations: $\mathrm{IV}=$ intravenous, $\mathrm{Rx}=$ therapy, $\mathrm{MD}=$ medical doctor, $\mathrm{PT}=$ physical therapy, $\mathrm{OT}=$ occupational therapy, $\mathrm{ST}=$ speech therapy)

Figure 4

component of the cost of managing MS $[4,48]$, but as practice patterns change there is an increased interest in avoiding hospitalization where clinically prudent, such as those due to bladder dysfunction by improving self care [48] or by utilizing alternative sites of care, such as observation units or home care. Our study suggests that the cost of managing a relapse will typically increase over six fold if inpatient care is provided, compared with a relapse that can be managed with IV steroids as an outpatient.

When assessing chronic clinical conditions in the US, Medicare is often the payer of interest given the usual age distribution of chronically ill patients; however, patients diagnosed with MS are typically younger than 65 years old, and so have insurance coverage from a variety of health care service plans. As would be expected for patients diagnosed with MS, most of the inpatient (90\%) and ER cases (93\%) were younger than 65 years old. Even so, Medicare was the primary payer for $39 \%$ of the inpatient cases and $23 \%$ of ER cases. Managed care plans paid for over a quarter of the inpatient cases $(28 \%)$, and for almost a third of the ER cases (32\%). These figures are consistent with a survey by the National Multiple Sclerosis Society, which indicated that while Medicare insured the majority of respondents, at least a quarter held private insurance[4]. Given these figures, although Medicare does 
have a larger than might be expected role in the coverage for this younger group of patients, economic concerns related to MS relapse management are likely to be equally germane to MCOs.

Scarce information was found in the literature that could shed light on the management cost of relapse in the US by level of health care needs. Most of the published studies from the US and elsewhere are cost of illness studies and include indirect costs which for the most part combine all types of MS into one group for reporting purposes so teasing out the cost of managing a relapse is not possible [37]. Other published sources, often cited in reports about MS, present information on studies that are not necessarily representative of an MS "real world" populations. For example, there are references in the literature to a health care cost study done on veterans in the US [48]. While this study provides valuable information, its relevance to the MS population as a whole and a managed care population in particular is questionable as $89 \%$ of the participants were male and only $13 \%$ of the patients had relapsingremitting MS. Prevalence data of MS in the US show that $73 \%$ of those with the condition are female [37]. The results of our data analysis support this in that the majority of formal care seekers were female at all locations of care in our study. This analysis, which uses an approach heretofore not taken in calculating the cost of managing a relapse, presents a detailed picture of possible management scenarios for relapse in actual practice settings.

In this study, the cost profiles developed for inpatient care, emergency room visits, nursing home stays and physician visits were all based on data collected from patients with a recorded diagnosis of MS. MS has been allocated a specific ICD-9 code, and this allowed selection of the cases with multiple sclerosis from the various databases used in these analyses. Unfortunately, the ICD-9 code did not allow us to differentiate between cases with different types of MS; therefore, it is unknown how many cases had the relapsing-remitting or progressive forms of the disease. Cases were excluded where the admission or the visit was primarily for depression or other mood disorders. In this analysis, these were considered to be related more to the consequences of living with multiple sclerosis than a relapse [37].

A key cost component of the medium intensity management level arises from administering a course of methylprednisolone infusions in the out patient setting. As would be expected, the cost estimated for home infusion therapy is substantially lower, approximately one quarter of the cost of providing this care as a hospital day case. One important limitation of our study is that whilst these are credible estimates of the cost of providing the infusions in each setting, information about the proportion of patients receiving these infusions as a day case was not available to us. The overall cost estimate for the medium management level assumed the minority (25\%) receives the infusions as a day case rather than home infusions, and this is conservative based on known insurance coverage restrictions [47] and personal communication with staff of several US based MS clinics. It is also likely that the portion of the costs contributed by testing is a conservative estimate. The low rates of diagnostic and laboratory tests noted in the NAMCS data may be more reflective of under coding diagnostic tests than the level of actual utilization.

The average wholesale price (AWP) was used as the basis for estimating the outpatient symptom-related medication profile. Although the AWP is not representative of the cost that most purchasers or users pay for a drug, it was selected as the reference cost to provide a common, understandable baseline from which a comparison can be made for a given circumstance. This approach was taken rather than base the analysis on any one organization's discounted rate; as such an estimate would not be representative past the environs of the selected organization.

It has been noted that patients with chronic illnesses adapt to their level of disability and learn to accommodate changes in physical condition without seeking formal care even when a symptom may be termed as "bothersome"[32]. Thus, not every mild relapse will generate costs even at the low management level. Nevertheless, based on our analyses of the NAMCS data, once patients attend their physician for an acute MS-related problem, almost two-thirds will return for a follow-up visit.

The development of disease modifying agents in recent years has altered the clinical management of this illness. The current health care environment requires decisionmakers to consider the fiscal, as well as the clinical consequences of therapy (i.e. cost-effectiveness analyses) when allocating limited health care dollars. The cost estimates for a relapse episode based on escalating levels of management presented in this paper provide valuable inputs for decision-makers and researchers seeking to evaluate and understand the economic, as well as the clinical implications of disease modifying drugs for MS.

\section{Conclusions}

This study provides decision-makers costs heretofore unknown based on current management practices. This analysis has shown that there is a cost to a relapse regardless of the level of management once formal care is sought. 
Clearly and not surprisingly, site of care will affect the cost of a relapse, even amongst those treated as outpatients. For patients who are initially seen in the ER, the cost of the medium level of management is estimated to increase by almost a third when compared with those seen initially by their physician. Policies or standardized protocols of care that lead to an increase in the proportion of patients receiving steroid infusions at home or in another ambulatory setting, or those that encourage attending their usual care physician rather than visiting the ER when clinically appropriate, would therefore have a substantial impact on the overall cost estimated for a relapse episode.

\section{Competing Interests}

Caro Research, of which Judith A. O'Brien and J. Jaime Caro are shareholders, received a grant from Serono International SA, which provided funding for portions of the study. They were allowed to review and comment on this manuscript but were explicitly forbidden from exerting any editorial control. The authors are employees of Caro Research.

\section{Authors' Contributions}

JAO conceived of and designed the study, performed the analysis, and participated in drafting the manuscript. AW contributed to the design of the study and drafted the manuscript. AP conducted and reported the analyses. JC participated in the conception and design of the study. All authors read and approved the final manuscript.

\section{Acknowledgements}

This work was funded in part by an unrestricted grant from Serono International SA

\section{References}

I. National Institute of Neurological Disorders and Stroke. Multiple Sclerosis: Hope Through Research NIH 2002 [http:// www.ninds.nih.gov/health and medical/pubs/multiple sclerosis.htm].

2. Runmarker B and Andersen O: Prognostic factors in a multiple sclerosis incidence cohort with twenty-five years of followup. Brain 1993, I16: I 7-164.

3. Weinshenker BG, Bass B, Rice GP, Noseworthy J, Carriere W, Baskerville J and Ebers GC: The natural history of multiple sclerosis: a geographically based study. 2. Predictive value of the early clinical course. Brain 1989, I | 2: |4| 9- | 428.

4. Whetten-Goldstein K, Sloan FA, Goldstein LB and Kulas ED: A comprehensive assessment of the cost of multiple sclerosis in the United States. Multiple Sclerosis 1998, 4:419-425.

5. Lacy JR, Stuart WH, Jacobs LD, Vartanian T, Phillips JT and Greenstein Jl: Registry of Multiple Sclerosis Treatment and Care (MSTRAC) Patient and Physician Characteristics. Int J MS Care 2000, 2(4): [http://www.mscare.com/a0012/page 04.htm].

6. Hauser SL and Goodkin DE: Multiple sclerosis and other demyelinating diseases. In: Harrison's Principles of Medicine 15th edition. McGraw-Hill Companies Inc.; 200I:2452-246I.

7. Scott TF, Schramke CJ, Novero J and Chieffe C: Short-term prognosis in early relapsing-remitting multiple sclerosis. Neurology 2000, 55:689-693.

8. California 1999 Discharge Data (Version A). Office of Statewide Health Planning and Development.

9. Florida 1999 Hospital Patient Data File. State of Florida, Agency for Health Care Administration, State Center for Health Statistics .
10. Massachusetts Fiscal Year 1999 Acute Hospital Case Mix Data Base, Level III. Massachusetts Division of Health Care Finance and Policy.

II. Maryland 1999 Inpatient Public Use File. St. Paul Computer Center, Inc .

12. Washington 1999 CHARS (Comprehensive Hospital Abstract Reporting System) Public Data File. Washington State Department of Health, Office of Hospital and Patient Data .

13. Annual Physician Fee Schedule Payment Amount File Health Care Financing Agency 2002 [http://www.cms.hhs.gov/providers/]. Accessed November 2001

14. Veririlli DK and Zuckerman S: Health Care Financing Review Spring. 1996, I7(3):161-170.

15. 200 I Florida Medicaid physician fee schedule [http:// www.fdhc.state.fl.us/Medicaid/index.shtml]

16. Foundation for Healthy Communities. Specialty Hospital Standard Reports, 1999. New Hampshire Hospital Association 2001.

17. Foundation for Healthy Communities. Specialty Hospital Standard Reports, 1998. New Hampshire Hospital Association 2000.

18. Foundation for Healthy Communities. Specialty Hospital Standard Reports, 1997. New Hampshire Hospital Association 1999.

19. Foundation for Healthy Communities. Specialty Hospital Standard Reports, 1996. New Hampshire Hospital Association 1998.

20. Medicare and Medicaid Statistical Supplement, 2000. Health Care Finance Review. U.S. Dept. of Health and Human Services. HCFA Office of Strategic Planning, Baltimore 200I.

21. Minnesota Case Mix Research Database. Quality Assurance and Review Program, Minnesota Department of Health 1998.

22. The Guide to the Nursing Home Industry, 200I. HCIASachs, L.L.C., Baltimore, MD and Arthur Andersen, L.L.P., Hartford, CT 2000.

23. Manton KG, Cornelius ES and Woodbury MA: Nursing home residents: a multivariate analysis of their medical, behavioral, psychosocial, and service use characteristics. J Gerontol A Biol Sci Med Sci 1995, 50(5):M242-M25I.

24. Utah Emergency Department Encounter Database, 1999 Public-Use Data File. Bureau of Emergency Medical Services, Utah Department of Health

25. Massachusetts Outpatient Observation Case Mix and Charge Data, Fiscal Year 1999. Massachusetts Division of Health Care Finance and Policy

26. Florida Medicaid Outpatient Database, 1999. Medicaid Program Analysis, State of Florida, Agency for Health Care Administration.

27. National Ambulatory Medical Care Survey National Center for Health Statistics, Washington, DC 1997 [http://www.cdc.gov/nchs/ about/major/ahcd/ahcd I.htm].

28. National Ambulatory Medical Care Survey National Center for Health Statistics, Washington, DC 1998 [http://www.cdc.gov/nchs/ about/major/ahcd/ahcdl.htm].

29. National Ambulatory Medical Care Survey National Center for Health Statistics, Washington, DC 1999 [http://www.cdc.gov/nchs/ about/major/ahcd/ahcdl.htm].

30. Parkin D, McNamee P, Jacoby A, Miller P, Thomas S and Bates D: A cost-utility analysis of interferon beta for multiple sclerosis. Health Technology Assessment 1998, 2(4):27-29.

31. Tremlett HL, Luscombe DK and Wiles CM: Use of corticosteroids in multiple sclerosis by consultant neurologists in the United Kingdom. J Neurol Neurosurg Psychiatry 1998, 65:362-365.

32. Vickrey BG, Shatin D, Wolf SM, Myers LW, Belin TR, Hanson RA, Shapiro MF, Beckstrand M, Edmonds ZV, Delrahim S and Ellison GW: Management of Multiple Sclerosis across managed care and fee-for-service systems. Neurology 2000, 55:134|-1349.

33. Rudick RA, Cohen JA, Weinstock-Guttman B, Kinkel RP and Ransohoff : Management of Multiple Sclerosis. N Engl J Med 1997, 337(22): 1604-1611.

34. The APG Handbook. HCIA Inc., Baltimore, MD and $3 M$ Health Care, Wallingford, CT 1997.

35. National Ambulatory Medical Care Survey National Center for Health Statistics, Washington, DC 1998 [http://www.cdc.gov/nchs/ about/major/ahcd/ahcd I.htm].

36. I999 National Ambulatory Medical Care Survey Data File Documentation National Center for Health Statistics, Washington, DC [http://www.cdc.gov/nchs/about/major/ahcd/ahcdI.htm].

37. Minden SL, Marder WD, Harrold LN and Dor A: Multiple Sclerosis, A Statistical Portrait. A Compendium of Data, Demographics, Disability and Health Services Utilization in the United States. Prepared for the National Multiple Sclerosis Society by AbtAssociates, Inc 1993. 
38. American Medical Association: Physicians' Current Procedural Terminology. Chicago, American Medical Association $200 \mathrm{I}$.

39. Grima DT, Torrance GW, Francis G, Rice G, Rosner AJ and Lafortune $\mathrm{L}$ : Cost and health quality of life consequences of multiple sclerosis. Multiple Sclerosis 2000, 6:91-98.

40. 2001 Drug Topics Red Book. Montvale, NJ, Medical Economics 1999.

4I. Beck RW, Cleary PA, Anderson MK, Keltner JL, Shults WT, Kaufman DI, Buckley EG, Corbett JJ, Kupersmith MJ, Miller NR, Savino PJ, Guy JR, Trobe JD, McCrary JA, Smith CH, Brodsky MC, Goodwin JA, Atwell CW and the Optic Neuritis Study Group: A Randomized, Clinical Trial of Corticosteroids in the Treatment of Acute Optic Neuritis. N Engl J Med 1992, 326(9):58I-588.

42. Wen LK, Lawson KA and Khan ZM: A Survey of Operational Costs Incurred by Home Infusion Pharmacies. Infusion 1997:44-51.

43. Bureau of Labor Statistics 1997 [http://www.bls.gov/cpi/ home.htm].

44. Weinshenker BG, Bass B, Rice GP, Noseworthy J, Carriere W, Baskerville J and Ebers GC: The natural history of multiple sclerosis: a geographically based study. I. Clinical course and disability. Brain 1989, I I2:133-146.

45. Confavreux $\mathrm{C}$, Vukusic $\mathrm{S}$, Moreau $\mathrm{T}$ and Adeleine P: Relapses and Progression in Multiple Sclerosis. N Engl J Med 2000, 343(20): 1430- I 438.

46. Burks J: Multiple Sclerosis Care: An Integrated Disease-Management Model. J of Spinal Cord Medicine 1998, 2 I (2): I I3-II 6.

47. Coverage Policy Bulletins No. 0264: Multiple Sclerosis Treatments Aetna Insurance Company [http://www.aetna.com/cpb/ data/CPBA0264.html].

48. Bourdette DN, Prochazka AV, Mitchell W, Licari P and Burks ]: Health Care Costs of Veterans with Multiple Sclerosis: Implications for the Rehabilitation of MS. Arch Phys Med Rehabil 1993, 74:26-3I.

\section{Pre-publication history}

The pre-publication history for this paper can be accessed here:

http://www.biomedcentral.com/1472-6963/3/17/prepub http.//www.bic 